Supplement of

\title{
Retrieval of UV-visible aerosol absorption using AERONET and OMI-MODIS synergy: spatial and temporal variability across major aerosol environments
}

Vinay Kayetha et al.

Correspondence to: Vinay Kayetha (vinay.k.kayetha@nasa.gov)

The copyright of individual parts of the supplement might differ from the article licence. 


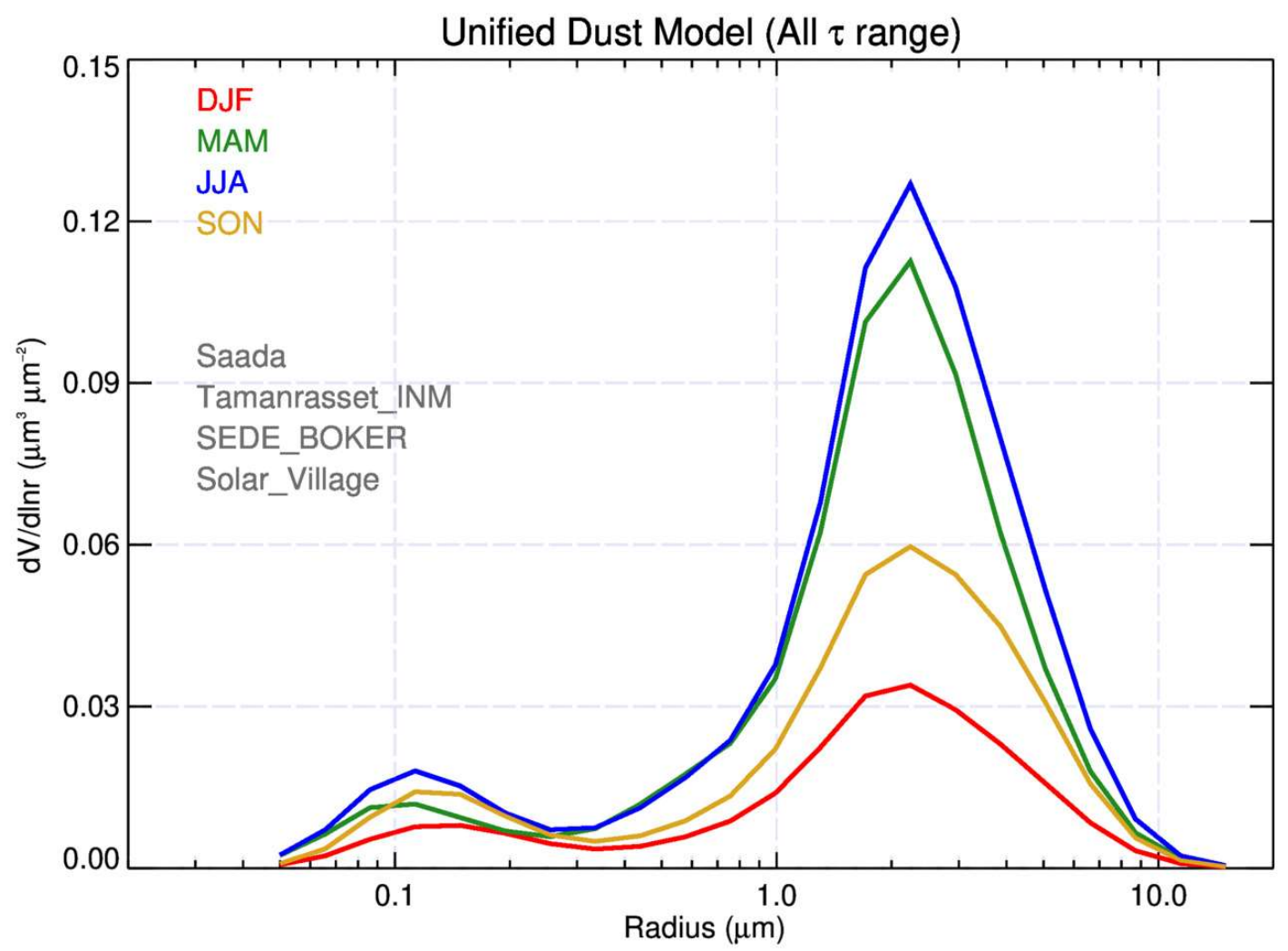

3

4

5 Figure S1: Seasonal climatology of particle size distributions derived from AERONET 6 Dust dominated sites for all $\tau$ observations. 
All $\tau$ range

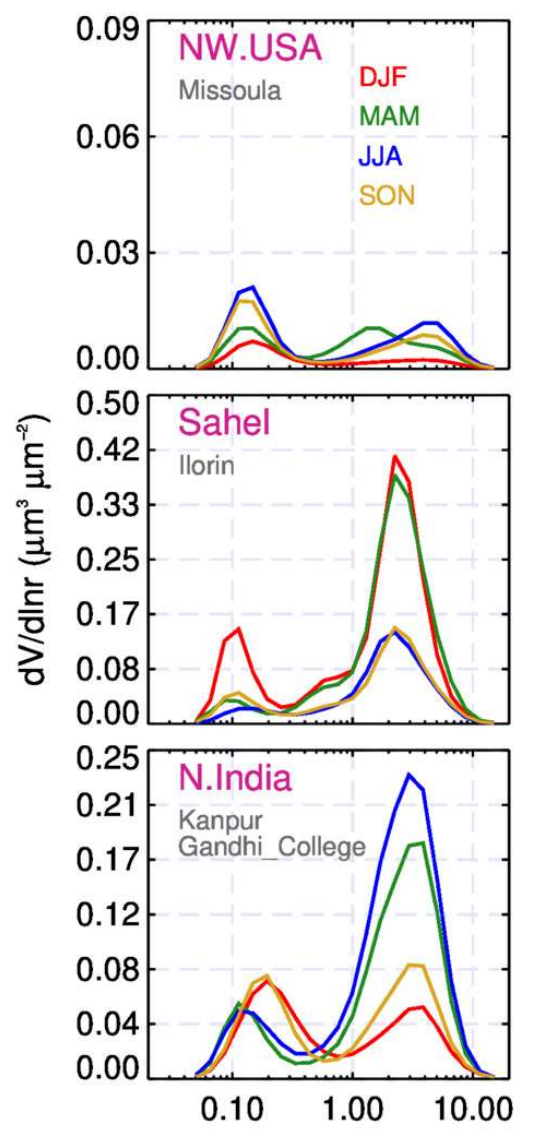

8
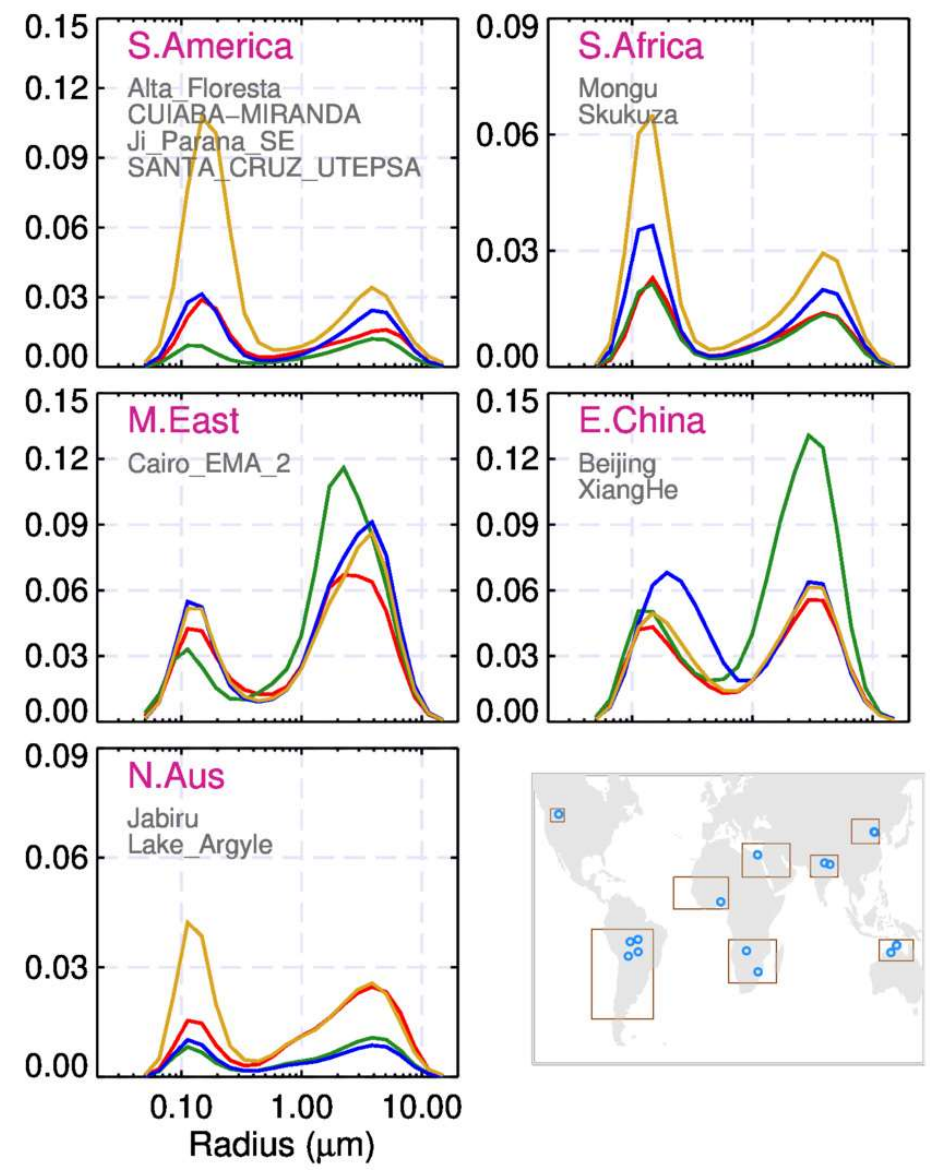

10 Figure S2: Seasonal climatology of particle size distributions derived from AERONET 11 Smoke dominated sites for all $\tau$ observations. 


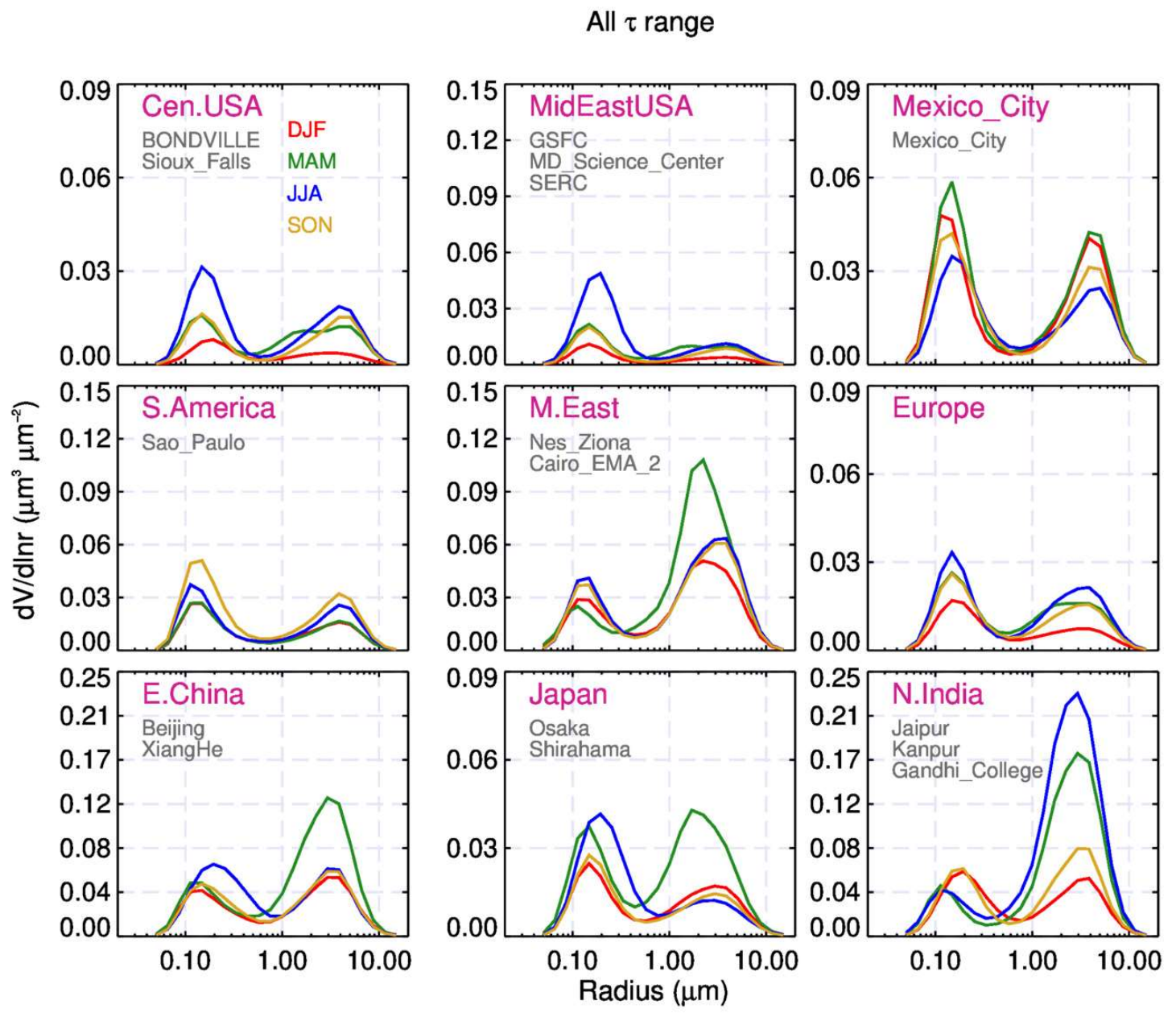

13

14

15 Figure S3: Seasonal climatology of particle size distributions derived from AERONET

16 Urban aerosols dominated sites for all $\tau$ observations.

17 
Table S1: Retrieved seasonal average SSA at 340, 354, 388, 466 and $646 \mathrm{~nm}$ and AAE at UV-Visible wavelength pairs for Carbonaceous, Dust, and Urban aerosols with observations $\tau_{440}>0.4$ over individual AERONET sites from $2005-2016$. Abbreviations used: AType is the aerosol type, NDays is the number of independent days, NObs is the number of observations.

\begin{tabular}{|c|c|c|c|c|c|c|c|c|c|c|c|c|c|c|c|}
\hline & \multirow[t]{2}{*}{ A.Type } & \multirow[t]{2}{*}{ Season } & \multirow[t]{2}{*}{ N.days } & \multicolumn{2}{|c|}{ N. Obs } & \multirow[t]{2}{*}{$\tau_{440}$} & \multirow[t]{2}{*}{$\alpha_{440-870}$} & \multicolumn{5}{|c|}{ Average SSA } & \multicolumn{3}{|c|}{ Average Abs.AE } \\
\hline & & & & OMI & MODIS & & & $340 \mathrm{~nm}$ & $354 \mathrm{~nm}$ & $388 \mathrm{~nm}$ & $466 \mathrm{~nm}$ & $646 \mathrm{~nm}$ & 354_388 & $466 \_646$ & $340 \_646$ \\
\hline \multicolumn{16}{|l|}{ NW. USA } \\
\hline \multirow{3}{*}{ Missoula } & Carbon. & JJA & 5 & 9 & 51 & 1.2302 & 1.78 & 0.887 & 0.903 & 0.913 & 0.936 & 0.904 & 3.976 & 1.037 & 1.705 \\
\hline & Urban & JJA & 10 & 26 & 83 & 0.6967 & 1.91 & 0.872 & 0.876 & 0.871 & 0.872 & 0.847 & 1.648 & 1.322 & 1.667 \\
\hline & Urban & SON & 8 & 21 & 60 & 0.5554 & 1.96 & 0.845 & 0.842 & 0.826 & 0.865 & 0.829 & 0.967 & 1.470 & 1.747 \\
\hline Rimrock & Urban & JJA & 6 & 38 & 25 & 0.5425 & 1.96 & 0.880 & 0.893 & 0.895 & 0.894 & 0.866 & 2.470 & 0.722 & 1.894 \\
\hline \multicolumn{16}{|l|}{ Central USA } \\
\hline Sioux_Falls & Urban & JJA & 8 & 40 & 96 & 0.4628 & 1.51 & 0.929 & 0.946 & 0.953 & 0.942 & 0.879 & 5.892 & -0.821 & 0.898 \\
\hline BONDVILLE & Urban & JJA & 12 & 51 & 127 & 0.4881 & 1.84 & 0.942 & 0.943 & 0.944 & 0.935 & 0.890 & 3.494 & -0.153 & 1.116 \\
\hline \multicolumn{16}{|l|}{ MidEastern USA } \\
\hline \multirow[t]{2}{*}{ GSFC } & Urban & MAM & 8 & 35 & 67 & 0.4907 & 1.51 & 0.930 & 0.947 & 0.951 & 0.943 & 0.941 & 3.034 & 1.717 & 1.598 \\
\hline & Urban & JJA & 9 & 46 & 23 & 0.4754 & 1.77 & 0.956 & 0.961 & 0.971 & 0.950 & 0.922 & 6.961 & 0.242 & 1.493 \\
\hline \multirow[t]{2}{*}{ MD_Science_Centr } & Urban & MAM & 8 & 52 & 62 & 0.4909 & 1.77 & 0.945 & 0.951 & 0.956 & 0.954 & 0.896 & 3.049 & -1.196 & 0.802 \\
\hline & Urban & JJA & 8 & 44 & 64 & 0.5245 & 1.89 & 0.931 & 0.937 & 0.947 & 0.919 & 0.868 & 6.328 & 0.025 & 1.560 \\
\hline Wallops & Urban & JJA & 8 & 19 & 17 & 0.6376 & 1.78 & 0.897 & 0.898 & 0.895 & 0.892 & 0.848 & 2.793 & 1.084 & 0.894 \\
\hline \multicolumn{16}{|l|}{ Central America } \\
\hline \multirow{3}{*}{ Mexico_City } & Urban & DJF & 53 & 223 & 255 & 0.5651 & 1.7 & 0.887 & 0.886 & 0.888 & 0.858 & 0.845 & 2.935 & 1.967 & 1.204 \\
\hline & Urban & MAM & 30 & 60 & 76 & 0.6873 & 1.68 & 0.904 & 0.910 & 0.916 & 0.865 & 0.832 & 3.821 & 1.182 & 0.948 \\
\hline & Urban & SON & 6 & 13 & 13 & 0.4738 & 1.67 & 0.882 & 0.873 & 0.863 & 0.852 & 0.805 & 0.843 & 0.437 & 0.850 \\
\hline \multicolumn{16}{|l|}{ South America } \\
\hline \multirow{3}{*}{ Alta_Floresta } & Carbon. & JJA & 10 & 66 & 94 & 0.8591 & 1.9 & 0.901 & 0.905 & 0.920 & 0.932 & 0.910 & 4.590 & 1.287 & 1.766 \\
\hline & Carbon. & SON & 10 & 50 & 155 & 1.0049 & 1.81 & 0.925 & 0.929 & 0.943 & 0.941 & 0.917 & 4.472 & 1.772 & 1.722 \\
\hline & Urban & SON & 7 & 15 & 66 & 0.623 & 1.69 & 0.919 & 0.934 & 0.939 & 0.932 & 0.878 & 2.041 & 0.650 & 0.811 \\
\hline \multirow{4}{*}{ CUIABA-MIRANDA } & Carbon. & JJA & 6 & 40 & 61 & 0.6997 & 1.75 & 0.883 & 0.889 & 0.912 & 0.900 & 0.870 & 4.959 & 1.118 & 2.053 \\
\hline & Carbon. & SON & 23 & 164 & 283 & 0.9377 & 1.76 & 0.900 & 0.907 & 0.919 & 0.914 & 0.880 & 3.711 & 0.831 & 1.359 \\
\hline & Urban & JJA & 7 & 36 & 103 & 0.5579 & 1.77 & 0.876 & 0.877 & 0.880 & 0.866 & 0.823 & 2.141 & 0.962 & 1.321 \\
\hline & Urban & SON & 27 & 213 & 446 & 0.5347 & 1.76 & 0.906 & 0.917 & 0.923 & 0.916 & 0.875 & 3.809 & 0.875 & 1.346 \\
\hline Rio_Branco & Urban & JJA & 8 & 47 & 127 & 0.5313 & 1.83 & 0.898 & 0.903 & 0.909 & 0.930 & 0.887 & 2.615 & 0.735 & 1.628 \\
\hline
\end{tabular}




\begin{tabular}{|c|c|c|c|c|c|c|c|c|c|c|c|c|c|c|c|}
\hline & Urban & SON & 8 & 57 & 117 & 0.5381 & 1.72 & 0.915 & 0.929 & 0.937 & 0.934 & 0.883 & 4.427 & 0.354 & 1.154 \\
\hline \multirow[t]{2}{*}{ Ji_Parana_SE } & Carbon. & JJA & 7 & 23 & 42 & 0.8592 & 1.83 & 0.920 & 0.922 & 0.930 & 0.927 & 0.914 & 3.177 & 1.790 & 1.687 \\
\hline & Urban & JJA & 5 & 39 & 25 & 0.4874 & 1.76 & 0.893 & 0.902 & 0.908 & 0.923 & 0.893 & 2.494 & 1.358 & 1.980 \\
\hline \multirow[t]{2}{*}{ SANTA_CRUZ_UTE } & Carbon. & SON & 9 & 23 & 79 & 0.7882 & 1.72 & 0.899 & 0.908 & 0.929 & 0.935 & 0.907 & 5.756 & 0.781 & 1.650 \\
\hline & Urban & SON & 14 & 78 & 225 & 0.5636 & 1.67 & 0.889 & 0.902 & 0.901 & 0.912 & 0.879 & 1.770 & 0.860 & 1.749 \\
\hline \multirow[t]{2}{*}{ Sao_Paulo } & Urban & JJA & 14 & 66 & 158 & 0.5026 & 1.45 & 0.876 & 0.871 & 0.856 & 0.853 & 0.837 & 0.413 & 1.400 & 0.967 \\
\hline & Urban & SON & 10 & 75 & 86 & 0.5175 & 1.54 & 0.898 & 0.908 & 0.916 & 0.910 & 0.880 & 3.242 & 0.900 & 1.496 \\
\hline \multicolumn{16}{|l|}{ Europe } \\
\hline \multirow[t]{2}{*}{ Avignon } & Mixt. & JJA & 5 & 29 & 64 & 0.5116 & 0.56 & 0.909 & 0.925 & 0.938 & 0.920 & 0.853 & 3.305 & -1.722 & -0.295 \\
\hline & Urban & JJA & 7 & 41 & 53 & 0.5099 & 1.6 & 0.927 & 0.938 & 0.945 & 0.929 & 0.861 & 3.150 & 0.118 & 0.538 \\
\hline Barcelona & Mixt. & JJA & 6 & 14 & 30 & 0.5231 & 0.41 & 0.899 & 0.906 & 0.920 & 0.938 & 0.868 & 2.442 & -2.070 & 0.271 \\
\hline Burjassot & Mixt. & JJA & 14 & 73 & 71 & 0.5031 & 0.67 & 0.900 & 0.906 & 0.919 & 0.933 & 0.881 & 3.480 & -0.897 & 0.640 \\
\hline \multirow{3}{*}{ Carpentras } & Mixt. & JJA & 6 & 38 & 128 & 0.4583 & 0.56 & 0.894 & 0.909 & 0.923 & 0.929 & 0.849 & 2.303 & -2.258 & -0.041 \\
\hline & Urban & MAM & 5 & 12 & 68 & 0.4639 & 1.41 & 0.912 & 0.932 & 0.925 & 0.882 & 0.847 & 0.294 & 1.014 & 0.668 \\
\hline & Urban & JJA & 8 & 41 & 106 & 0.4641 & 1.7 & 0.929 & 0.941 & 0.947 & 0.902 & 0.822 & 4.642 & 0.318 & 0.642 \\
\hline FORTH_CRETE & Mixt. & MAM & 7 & 31 & 33 & 0.5814 & 0.35 & 0.896 & 0.911 & 0.932 & 0.942 & 0.940 & 4.240 & -0.441 & 1.323 \\
\hline \multirow{3}{*}{ IMS-METU-ERDEM } & Mixt. & MAM & 10 & 49 & 37 & 0.636 & 0.37 & 0.893 & 0.909 & 0.920 & 0.921 & 0.885 & 2.510 & -0.518 & 0.206 \\
\hline & Mixt. & JJA & 11 & 44 & 28 & 0.558 & 0.89 & 0.889 & 0.897 & 0.902 & 0.894 & 0.888 & 1.924 & 1.298 & 0.875 \\
\hline & Urban & JJA & 18 & 39 & 32 & 0.5094 & 1.45 & 0.920 & 0.930 & 0.937 & 0.870 & 0.874 & 3.723 & 2.253 & 0.847 \\
\hline \multirow[t]{2}{*}{ Ispra } & Urban & JJA & 7 & 16 & 32 & 0.5968 & 1.59 & 0.947 & 0.958 & 0.961 & 0.930 & 0.898 & 4.259 & 0.620 & 0.407 \\
\hline & Urban & SON & 6 & 8 & 45 & 0.5364 & 1.5 & 0.885 & 0.904 & 0.889 & 0.934 & 0.869 & 0.430 & -1.554 & 1.094 \\
\hline \multirow[t]{2}{*}{ Lecce_University } & Mixt. & JJA & 25 & 141 & 104 & 0.5095 & 0.52 & 0.902 & 0.912 & 0.931 & 0.947 & 0.914 & 4.127 & -1.275 & 0.873 \\
\hline & Urban & JJA & 16 & 75 & 81 & 0.4769 & 1.75 & 0.928 & 0.934 & 0.938 & 0.941 & 0.902 & 2.819 & 0.540 & 1.245 \\
\hline \multirow[t]{2}{*}{ Lille } & Urban & MAM & 5 & 42 & 127 & 0.4655 & 1.63 & 0.916 & 0.935 & 0.945 & 0.936 & 0.934 & 4.607 & 2.322 & 1.963 \\
\hline & Urban & JJA & 6 & 31 & 99 & 0.4739 & 1.6 & 0.922 & 0.938 & 0.945 & 0.937 & 0.895 & 4.022 & -0.166 & 1.283 \\
\hline Minsk & Urban & MAM & 5 & 20 & 118 & 0.4652 & 1.64 & 0.926 & 0.949 & 0.957 & 0.923 & 0.907 & 4.330 & 1.177 & 1.453 \\
\hline \multirow{5}{*}{ Modena } & Mixt. & MAM & 7 & 29 & 108 & 0.5461 & 0.92 & 0.907 & 0.921 & 0.932 & 0.921 & 0.868 & 3.616 & -0.797 & 0.586 \\
\hline & Mixt. & JJA & 14 & 90 & 331 & 0.5164 & 0.96 & 0.906 & 0.910 & 0.917 & 0.945 & 0.884 & 2.641 & -1.540 & 0.679 \\
\hline & Urban & MAM & 24 & 156 & 614 & 0.4805 & 1.54 & 0.918 & 0.925 & 0.927 & 0.932 & 0.892 & 2.243 & 0.377 & 0.956 \\
\hline & Urban & JJA & 36 & 197 & 738 & 0.5306 & 1.61 & 0.934 & 0.941 & 0.945 & 0.924 & 0.869 & 3.880 & 0.053 & 0.543 \\
\hline & Urban & SON & 10 & 32 & 245 & 0.4795 & 1.53 & 0.900 & 0.924 & 0.925 & 0.933 & 0.906 & 3.161 & 0.361 & 1.199 \\
\hline \multirow[t]{2}{*}{ Moldova } & Mixt. & MAM & 6 & 46 & 186 & 0.4975 & 0.66 & 0.880 & 0.902 & 0.918 & 0.952 & 0.906 & 2.829 & -1.483 & 0.974 \\
\hline & Urban & JJA & 14 & 64 & 227 & 0.4817 & 1.74 & 0.924 & 0.929 & 0.924 & 0.931 & 0.909 & 1.258 & 1.304 & 1.505 \\
\hline \multirow[t]{2}{*}{ Moscow_MSU_MO } & Urban & MAM & 8 & 84 & 173 & 0.4914 & 1.5 & 0.909 & 0.926 & 0.933 & 0.934 & 0.891 & 5.095 & 0.258 & 1.054 \\
\hline & Urban & JJA & 11 & 72 & 195 & 0.4724 & 1.56 & 0.908 & 0.922 & 0.923 & 0.932 & 0.873 & 2.505 & -0.362 & 1.163 \\
\hline
\end{tabular}

Page 5 of 10 


\begin{tabular}{|c|c|c|c|c|c|c|c|c|c|c|c|c|c|c|c|}
\hline \multirow[t]{2}{*}{ Palaiseau } & Urban & MAM & 6 & 30 & 68 & 0.4696 & 1.57 & 0.930 & 0.945 & 0.951 & 0.945 & 0.920 & 3.263 & 0.566 & 1.359 \\
\hline & Urban & JJA & 10 & 54 & 100 & 0.4723 & 1.66 & 0.917 & 0.924 & 0.928 & 0.944 & 0.889 & 3.252 & -0.676 & 1.508 \\
\hline \multirow{5}{*}{ Rome_Tor_Vergata } & Mixt. & MAM & 6 & 32 & 40 & 0.4835 & 0.46 & 0.911 & 0.930 & 0.944 & 0.936 & 0.872 & 3.772 & -1.960 & -0.202 \\
\hline & Mixt. & JJA & 11 & 90 & 193 & 0.4611 & 0.53 & 0.896 & 0.904 & 0.920 & 0.937 & 0.889 & 3.227 & -0.909 & 0.516 \\
\hline & Urban & MAM & 7 & 33 & 47 & 0.4659 & 1.45 & 0.914 & 0.930 & 0.932 & 0.896 & 0.861 & 2.055 & 0.985 & 0.983 \\
\hline & Urban & JJA & 19 & 70 & 164 & 0.4737 & 1.62 & 0.924 & 0.929 & 0.928 & 0.881 & 0.862 & 1.878 & 1.776 & 0.774 \\
\hline & Urban & SON & 7 & 36 & 104 & 0.5016 & 1.42 & 0.867 & 0.861 & 0.844 & 0.815 & 0.799 & 0.236 & 1.341 & 0.653 \\
\hline \multirow{4}{*}{ Thessaloniki } & Mixt. & MAM & 5 & 26 & 33 & 0.5258 & 1.01 & 0.913 & 0.931 & 0.938 & 0.930 & 0.916 & 3.282 & 0.912 & 1.021 \\
\hline & Mixt. & JJA & 13 & 119 & 189 & 0.5344 & 0.66 & 0.915 & 0.923 & 0.932 & 0.948 & 0.893 & 2.298 & -1.433 & 0.733 \\
\hline & Mixt. & SON & 6 & 47 & 121 & 0.4802 & 1.12 & 0.900 & 0.912 & 0.911 & 0.871 & 0.862 & 1.560 & 1.240 & 0.641 \\
\hline & Urban & JJA & 35 & 127 & 200 & 0.5144 & 1.64 & 0.935 & 0.939 & 0.941 & 0.914 & 0.905 & 3.557 & 1.920 & 0.904 \\
\hline Toulon & Mixt. & JJA & 5 & 30 & 40 & 0.491 & 0.81 & 0.899 & 0.922 & 0.930 & 0.931 & 0.895 & 2.698 & -0.272 & 0.903 \\
\hline \multicolumn{16}{|l|}{ Sahel } \\
\hline \multirow{8}{*}{ Banizoumbou } & Dust & DJF & 17 & 151 & 175 & 0.7599 & 0.13 & 0.931 & 0.935 & 0.946 & 0.958 & 0.971 & 2.374 & 1.876 & 1.777 \\
\hline & Dust & MAM & 153 & 1509 & 1399 & 0.7852 & 0.13 & 0.896 & 0.909 & 0.932 & 0.935 & 0.957 & 4.386 & 2.047 & 2.010 \\
\hline & Dust & JJA & 53 & 391 & 500 & 0.647 & 0.11 & 0.884 & 0.901 & 0.927 & 0.927 & 0.953 & 4.092 & 2.011 & 1.908 \\
\hline & Dust & SON & 19 & 164 & 82 & 0.5226 & 0.17 & 0.917 & 0.930 & 0.946 & 0.937 & 0.957 & 4.528 & 1.925 & 1.701 \\
\hline & Mixt. & DJF & 115 & 941 & 3078 & 0.675 & 0.47 & 0.909 & 0.913 & 0.925 & 0.937 & 0.932 & 2.752 & 0.491 & 1.499 \\
\hline & Mixt. & MAM & 67 & 669 & 943 & 0.6566 & 0.25 & 0.908 & 0.920 & 0.942 & 0.946 & 0.931 & 4.976 & -0.176 & 1.138 \\
\hline & Mixt. & JJA & 15 & 94 & 285 & 0.5056 & 0.37 & 0.879 & 0.897 & 0.914 & 0.928 & 0.908 & 4.501 & -0.206 & 1.200 \\
\hline & Mixt. & SON & 50 & 356 & 1166 & 0.5056 & 0.37 & 0.926 & 0.937 & 0.952 & 0.943 & 0.907 & 5.041 & -1.131 & 0.117 \\
\hline \multirow{7}{*}{ Dakar } & Dust & DJF & 21 & 117 & 128 & 0.6452 & 0.14 & 0.913 & 0.916 & 0.929 & 0.949 & 0.977 & 2.586 & 3.267 & 2.527 \\
\hline & Dust & MAM & 85 & 527 & 568 & 0.757 & 0.13 & 0.883 & 0.894 & 0.919 & 0.931 & 0.973 & 3.826 & 3.988 & 3.062 \\
\hline & Dust & JJA & 51 & 239 & 317 & 0.8007 & 0.1 & 0.867 & 0.883 & 0.913 & 0.932 & 0.971 & 4.174 & 3.326 & 2.696 \\
\hline & Dust & SON & 6 & 19 & 9 & 0.7943 & 0.1 & 0.903 & 0.915 & 0.931 & 0.937 & 0.958 & 2.714 & 1.704 & 1.720 \\
\hline & Mixt. & DJF & 63 & 366 & 423 & 0.5961 & 0.51 & 0.893 & 0.900 & 0.915 & 0.924 & 0.957 & 2.802 & 3.109 & 2.357 \\
\hline & Mixt. & MAM & 57 & 337 & 303 & 0.5534 & 0.34 & 0.894 & 0.905 & 0.927 & 0.938 & 0.970 & 4.268 & 3.633 & 2.649 \\
\hline & Mixt. & SON & 13 & 48 & 89 & 0.5509 & 0.46 & 0.911 & 0.915 & 0.930 & 0.915 & 0.895 & 3.734 & 0.002 & 0.729 \\
\hline \multirow{7}{*}{ IER_Cinzana } & Dust & DJF & 26 & 227 & 322 & 0.7313 & 0.13 & 0.906 & 0.909 & 0.921 & 0.938 & 0.969 & 1.789 & 3.185 & 2.501 \\
\hline & Dust & MAM & 93 & 899 & 969 & 0.8274 & 0.12 & 0.883 & 0.891 & 0.911 & 0.930 & 0.969 & 2.693 & 3.606 & 2.770 \\
\hline & Dust & JJA & 49 & 292 & 281 & 0.7129 & 0.09 & 0.868 & 0.884 & 0.915 & 0.939 & 0.973 & 3.705 & 3.707 & 3.049 \\
\hline & Dust & SON & 19 & 152 & 225 & 0.6054 & 0.13 & 0.898 & 0.909 & 0.921 & 0.935 & 0.959 & 1.808 & 2.021 & 1.438 \\
\hline & Mixt. & DJF & 93 & 731 & 1379 & 0.6492 & 0.49 & 0.894 & 0.896 & 0.906 & 0.920 & 0.949 & 1.834 & 2.606 & 2.252 \\
\hline & Mixt. & MAM & 44 & 325 & 388 & 0.5777 & 0.3 & 0.888 & 0.897 & 0.915 & 0.925 & 0.953 & 3.231 & 2.639 & 2.169 \\
\hline & Mixt. & JJA & 7 & 22 & 37 & 0.5117 & 0.37 & 0.922 & 0.936 & 0.953 & 0.935 & 0.941 & 4.496 & 1.374 & 2.257 \\
\hline
\end{tabular}

Page 6 of 10 


\begin{tabular}{|c|c|c|c|c|c|c|c|c|c|c|c|c|c|c|c|}
\hline & Mixt. & SON & 26 & 206 & 476 & 0.4916 & 0.34 & 0.922 & 0.932 & 0.942 & 0.941 & 0.917 & 4.056 & -0.354 & 0.596 \\
\hline \multirow{7}{*}{ Ilorin } & Dust & MAM & 7 & 93 & 134 & 1.1785 & 0.15 & 0.875 & 0.887 & 0.909 & 0.923 & 0.942 & 2.801 & 1.152 & 1.330 \\
\hline & Mixt. & DJF & 295 & 2797 & 8706 & 1.1725 & 0.68 & 0.888 & 0.891 & 0.897 & 0.903 & 0.901 & 1.501 & 0.780 & 1.095 \\
\hline & Mixt. & MAM & 63 & 537 & 821 & 0.8371 & 0.33 & 0.895 & 0.908 & 0.928 & 0.924 & 0.935 & 4.489 & 1.462 & 1.355 \\
\hline & Mixt. & SON & 38 & 345 & 854 & 0.6246 & 0.66 & 0.899 & 0.904 & 0.911 & 0.923 & 0.882 & 2.302 & -0.876 & 0.429 \\
\hline & Carbon. & DJF & 19 & 77 & 259 & 1.0556 & 1.33 & 0.866 & 0.863 & 0.867 & 0.872 & 0.866 & 1.676 & 1.252 & 1.366 \\
\hline & Urban & DJF & 37 & 259 & 1215 & 0.8462 & 1.3 & 0.866 & 0.864 & 0.861 & 0.857 & 0.859 & 1.078 & 1.465 & 1.279 \\
\hline & Urban & SON & 5 & 62 & 188 & 0.5491 & 1.31 & 0.866 & 0.870 & 0.872 & 0.855 & 0.842 & 1.660 & 1.318 & 1.167 \\
\hline \multirow{5}{*}{ Ouagadougou } & Dust & DJF & 6 & 110 & 229 & 1.3104 & 0.09 & 0.893 & 0.900 & 0.914 & 0.931 & 0.962 & 1.694 & 2.198 & 1.519 \\
\hline & Dust & MAM & 19 & 240 & 345 & 0.8722 & 0.13 & 0.895 & 0.903 & 0.921 & 0.925 & 0.969 & 2.722 & 3.495 & 1.981 \\
\hline & Dust & JJA & 11 & 72 & 127 & 0.9311 & 0.09 & 0.890 & 0.905 & 0.932 & 0.930 & 0.961 & 4.161 & 2.414 & 2.055 \\
\hline & Mixt. & DJF & 19 & 179 & 438 & 0.9063 & 0.36 & 0.906 & 0.908 & 0.917 & 0.937 & 0.962 & 1.679 & 2.593 & 2.415 \\
\hline & Mixt. & SON & 6 & 81 & 142 & 0.6574 & 0.33 & 0.926 & 0.933 & 0.941 & 0.933 & 0.938 & 2.979 & 1.276 & 0.716 \\
\hline \multirow{6}{*}{ Zinder_Airport } & Dust & MAM & 33 & 388 & 356 & 0.8138 & 0.13 & 0.883 & 0.895 & 0.916 & 0.941 & 0.981 & 3.005 & 4.551 & 3.582 \\
\hline & Dust & JJA & 26 & 206 & 123 & 0.652 & 0.09 & 0.873 & 0.892 & 0.923 & 0.948 & 0.987 & 4.586 & 5.759 & 3.986 \\
\hline & Mixt. & DJF & 22 & 207 & 259 & 0.5807 & 0.55 & 0.890 & 0.897 & 0.912 & 0.943 & 0.983 & 2.562 & 5.038 & 3.481 \\
\hline & Mixt. & MAM & 22 & 195 & 160 & 0.6094 & 0.27 & 0.879 & 0.890 & 0.909 & 0.953 & 0.981 & 2.410 & 4.547 & 3.543 \\
\hline & Mixt. & JJA & 6 & 47 & 28 & 0.5309 & 0.27 & 0.897 & 0.918 & 0.938 & 0.961 & 0.967 & 3.736 & 1.977 & 1.868 \\
\hline & Mixt. & SON & 15 & 87 & 181 & 0.5209 & 0.38 & 0.905 & 0.918 & 0.933 & 0.940 & 0.965 & 3.362 & 2.495 & 1.822 \\
\hline \multirow{8}{*}{ Agoufou } & Dust & DJF & 13 & 107 & 197 & 0.8096 & 0.11 & 0.923 & 0.926 & 0.939 & 0.952 & 0.968 & 2.477 & 1.980 & 1.894 \\
\hline & Dust & MAM & 86 & 905 & 975 & 0.79 & 0.12 & 0.899 & 0.910 & 0.930 & 0.935 & 0.958 & 3.533 & 2.021 & 1.858 \\
\hline & Dust & JJA & 68 & 560 & 890 & 0.6931 & 0.09 & 0.894 & 0.906 & 0.928 & 0.923 & 0.950 & 3.726 & 1.924 & 1.579 \\
\hline & Dust & SON & 18 & 140 & 192 & 0.625 & 0.12 & 0.899 & 0.913 & 0.934 & 0.941 & 0.956 & 4.159 & 1.565 & 1.708 \\
\hline & Mixt. & DJF & 33 & 329 & 927 & 0.6317 & 0.43 & 0.916 & 0.922 & 0.936 & 0.935 & 0.924 & 3.119 & 0.155 & 0.770 \\
\hline & Mixt. & MAM & 24 & 272 & 355 & 0.541 & 0.25 & 0.922 & 0.931 & 0.950 & 0.924 & 0.900 & 5.446 & -0.103 & 0.606 \\
\hline & Mixt. & JJA & 14 & 88 & 265 & 0.4565 & 0.24 & 0.897 & 0.909 & 0.927 & 0.938 & 0.908 & 4.032 & -0.856 & 0.246 \\
\hline & Mixt. & SON & 26 & 192 & 518 & 0.5014 & 0.37 & 0.929 & 0.940 & 0.955 & 0.949 & 0.913 & 5.103 & -1.286 & 0.113 \\
\hline \multicolumn{16}{|l|}{ Southern Africa } \\
\hline \multirow{4}{*}{ Mongu } & Carbon. & JJA & 25 & 156 & 328 & 0.7731 & 1.83 & 0.853 & 0.852 & 0.861 & 0.882 & 0.835 & 2.490 & 0.922 & 1.718 \\
\hline & Carbon. & SON & 21 & 182 & 447 & 1.0813 & 1.79 & 0.863 & 0.867 & 0.881 & 0.906 & 0.862 & 3.023 & 0.797 & 1.591 \\
\hline & Urban & JJA & 29 & 316 & 358 & 0.5136 & 1.85 & 0.868 & 0.868 & 0.869 & 0.905 & 0.853 & 1.899 & 0.910 & 2.122 \\
\hline & Urban & SON & 19 & 208 & 135 & 0.7031 & 1.71 & 0.879 & 0.885 & 0.889 & 0.933 & 0.873 & 2.147 & 0.050 & 1.783 \\
\hline \multirow{3}{*}{ Skukuza } & Carbon. & SON & 7 & 51 & 56 & 0.565 & 1.67 & 0.898 & 0.906 & 0.934 & 0.922 & 0.900 & 6.811 & 1.122 & 1.469 \\
\hline & Urban & JJA & 8 & 66 & 120 & 0.5346 & 1.59 & 0.894 & 0.900 & 0.904 & 0.922 & 0.903 & 2.949 & 0.750 & 1.622 \\
\hline & Urban & SON & 11 & 104 & 180 & 0.5055 & 1.6 & 0.902 & 0.914 & 0.933 & 0.880 & 0.847 & 4.635 & 1.188 & 1.428 \\
\hline
\end{tabular}

Page 7 of 10 


\begin{tabular}{|c|c|c|c|c|c|c|c|c|c|c|c|c|c|c|c|}
\hline \multirow[t]{2}{*}{ Mongu_Inn } & Urban & JJA & 14 & 44 & 70 & 0.6429 & 1.74 & 0.844 & 0.847 & 0.856 & 0.867 & 0.910 & 2.502 & 3.487 & 2.718 \\
\hline & Urban & SON & 27 & 141 & 354 & 0.9035 & 1.78 & 0.857 & 0.855 & 0.852 & 0.851 & 0.910 & 1.593 & 4.028 & 2.920 \\
\hline \multirow[t]{2}{*}{ Pretoria_CSIR-DPS } & Urban & JJA & 5 & 40 & 61 & 0.4985 & 1.5 & 0.864 & 0.871 & 0.884 & 0.914 & 0.881 & 2.837 & 0.995 & 1.984 \\
\hline & Urban & SON & 7 & 70 & 113 & 0.4302 & 1.48 & 0.868 & 0.873 & 0.883 & 0.908 & 0.874 & 2.935 & 1.261 & 1.740 \\
\hline \multicolumn{16}{|l|}{ Sahara } \\
\hline \multirow{4}{*}{ Saada } & Dust & JJA & 44 & 248 & 276 & 0.6492 & 0.13 & 0.881 & 0.897 & 0.928 & 0.946 & 0.980 & 4.974 & 4.251 & 2.950 \\
\hline & Dust & SON & 5 & 27 & 55 & 0.5454 & 0.13 & 0.892 & 0.902 & 0.930 & 0.942 & 0.970 & 4.912 & 3.758 & 2.766 \\
\hline & Mixt. & MAM & 8 & 55 & 127 & 0.607 & 0.29 & 0.877 & 0.894 & 0.912 & 0.943 & 0.945 & 2.618 & 0.681 & 1.620 \\
\hline & Mixt. & JJA & 43 & 245 & 218 & 0.5179 & 0.28 & 0.886 & 0.899 & 0.926 & 0.948 & 0.966 & 4.910 & 2.447 & 2.535 \\
\hline \multirow{5}{*}{ Tamanrasset_INM } & Dust & MAM & 22 & 159 & 239 & 0.7692 & 0.16 & 0.867 & 0.889 & 0.916 & 0.930 & 0.972 & 3.894 & 3.937 & 3.285 \\
\hline & Dust & JJA & 40 & 241 & 303 & 0.8026 & 0.1 & 0.873 & 0.890 & 0.917 & 0.930 & 0.972 & 3.900 & 4.002 & 3.074 \\
\hline & Dust & SON & 7 & 19 & 30 & 0.7351 & 0.1 & 0.877 & 0.893 & 0.918 & 0.939 & 0.979 & 3.728 & 4.490 & 2.839 \\
\hline & Mixt. & MAM & 16 & 128 & 175 & 0.5826 & 0.26 & 0.875 & 0.893 & 0.920 & 0.926 & 0.965 & 3.752 & 3.851 & 2.368 \\
\hline & Mixt. & JJA & 6 & 35 & 33 & 0.4613 & 0.29 & 0.851 & 0.867 & 0.893 & 0.916 & 0.960 & 2.825 & 3.820 & 2.651 \\
\hline \multicolumn{16}{|l|}{ Middle East } \\
\hline \multirow{4}{*}{ Nes_Ziona } & Mixt. & MAM & 14 & 90 & 72 & 0.4771 & 0.45 & 0.894 & 0.909 & 0.925 & 0.923 & 0.903 & 3.903 & -0.157 & 0.884 \\
\hline & Mixt. & JJA & 11 & 84 & 57 & 0.5146 & 0.87 & 0.918 & 0.927 & 0.940 & 0.928 & 0.926 & 3.866 & 1.133 & 1.515 \\
\hline & Mixt. & SON & 7 & 38 & 31 & 0.5544 & 0.98 & 0.901 & 0.914 & 0.926 & 0.941 & 0.929 & 2.922 & 0.180 & 1.613 \\
\hline & Urban & JJA & 5 & 28 & 19 & 0.5302 & 1.4 & 0.924 & 0.926 & 0.931 & 0.929 & 0.911 & 2.337 & 2.210 & 1.982 \\
\hline \multirow{3}{*}{ SEDE_BOKER } & Dust & MAM & 13 & 96 & 48 & 0.7777 & 0.1 & 0.896 & 0.911 & 0.940 & 0.960 & 0.984 & 5.712 & 3.639 & 3.202 \\
\hline & Mixt. & MAM & 9 & 54 & 21 & 0.5125 & 0.27 & 0.898 & 0.912 & 0.938 & 0.963 & 0.961 & 6.246 & 0.788 & 2.232 \\
\hline & Mixt. & JJA & 7 & 35 & 12 & 0.573 & 0.48 & 0.898 & 0.914 & 0.940 & 0.963 & 0.977 & 5.104 & 3.567 & 3.007 \\
\hline \multirow{6}{*}{ Solar_Village } & Dust & DJF & 9 & 53 & 57 & 0.6036 & 0.12 & 0.940 & 0.947 & 0.966 & 0.958 & 0.984 & 8.175 & 3.795 & 2.763 \\
\hline & Dust & MAM & 5 & 47 & 9 & 0.9347 & 0.06 & 0.901 & 0.912 & 0.938 & 0.971 & 0.991 & 3.967 & 4.090 & 4.290 \\
\hline & Dust & JJA & 21 & 187 & 222 & 0.9524 & 0.11 & 0.901 & 0.913 & 0.937 & 0.958 & 0.984 & 4.013 & 3.843 & 3.769 \\
\hline & Dust & SON & 7 & 31 & 34 & 0.832 & 0.12 & 0.910 & 0.921 & 0.947 & 0.960 & 0.986 & 5.614 & 4.659 & 3.813 \\
\hline & Mixt. & JJA & 28 & 201 & 80 & 0.6076 & 0.4 & 0.905 & 0.917 & 0.938 & 0.962 & 0.981 & 4.444 & 4.569 & 3.996 \\
\hline & Mixt. & SON & 9 & 63 & 47 & 0.493 & 0.51 & 0.918 & 0.929 & 0.946 & 0.961 & 0.979 & 3.629 & 3.803 & 2.505 \\
\hline \multirow{7}{*}{ Cairo_EMA_2 } & Dust & MAM & 5 & 14 & 16 & 0.9484 & 0.12 & 0.892 & 0.906 & 0.927 & 0.966 & 0.987 & 3.125 & 3.380 & 3.646 \\
\hline & Mixt. & DJF & 9 & 52 & 122 & 0.6635 & 0.78 & 0.889 & 0.898 & 0.904 & 0.908 & 0.902 & 1.693 & 1.562 & 1.426 \\
\hline & Mixt. & MAM & 82 & 588 & 568 & 0.5654 & 0.62 & 0.892 & 0.906 & 0.921 & 0.929 & 0.908 & 3.364 & 0.450 & 1.353 \\
\hline & Mixt. & JJA & 103 & 556 & 769 & 0.5125 & 0.89 & 0.892 & 0.901 & 0.913 & 0.932 & 0.901 & 2.843 & 0.309 & 1.288 \\
\hline & Mixt. & SON & 29 & 151 & 209 & 0.5258 & 0.88 & 0.881 & 0.890 & 0.898 & 0.918 & 0.895 & 2.294 & 0.474 & 1.599 \\
\hline & Carbon. & DJF & 5 & 8 & 14 & 0.6311 & 1.41 & 0.890 & 0.892 & 0.900 & 0.898 & 0.916 & 2.913 & 2.459 & 1.863 \\
\hline & Urban & DJF & 11 & 50 & 113 & 0.6341 & 1.34 & 0.881 & 0.883 & 0.876 & 0.860 & 0.874 & 1.216 & 2.333 & 1.775 \\
\hline
\end{tabular}




\begin{tabular}{|c|c|c|c|c|c|c|c|c|c|c|c|c|c|c|c|}
\hline & Urban & MAM & 15 & 78 & 98 & 0.5369 & 1.31 & 0.896 & 0.910 & 0.920 & 0.916 & 0.898 & 4.004 & 1.398 & 1.771 \\
\hline & Urban & JJA & 51 & 162 & 286 & 0.5246 & 1.3 & 0.890 & 0.899 & 0.903 & 0.904 & 0.868 & 2.150 & 0.740 & 1.308 \\
\hline & Urban & SON & 24 & 72 & 150 & 0.6005 & 1.33 & 0.878 & 0.884 & 0.884 & 0.904 & 0.894 & 1.668 & 1.871 & 1.969 \\
\hline \multicolumn{16}{|c|}{ NorthEastern Asia } \\
\hline \multirow{12}{*}{ Beijing } & Dust & MAM & 11 & 75 & 126 & 1.1352 & 0.1 & 0.882 & 0.907 & 0.923 & 0.930 & 0.950 & 2.954 & 1.559 & 1.445 \\
\hline & Mixt. & DJF & 23 & 133 & 605 & 0.8367 & 0.99 & 0.865 & 0.885 & 0.891 & 0.891 & 0.872 & 1.898 & 0.368 & 1.183 \\
\hline & Mixt. & MAM & 95 & 717 & 1920 & 0.8858 & 0.74 & 0.895 & 0.912 & 0.925 & 0.929 & 0.902 & 3.184 & -0.071 & 0.906 \\
\hline & Mixt. & JJA & 12 & 59 & 191 & 0.609 & 0.72 & 0.893 & 0.915 & 0.922 & 0.918 & 0.859 & 2.979 & -1.271 & 0.283 \\
\hline & Mixt. & SON & 17 & 81 & 507 & 0.6004 & 0.94 & 0.871 & 0.883 & 0.880 & 0.904 & 0.877 & 0.812 & 0.170 & 0.917 \\
\hline & Carbon. & DJF & 39 & 176 & 503 & 0.9813 & 1.34 & 0.874 & 0.887 & 0.898 & 0.906 & 0.889 & 2.834 & 0.931 & 1.638 \\
\hline & Carbon. & MAM & 10 & 31 & 72 & 1.073 & 1.36 & 0.895 & 0.907 & 0.915 & 0.918 & 0.912 & 2.488 & 1.332 & 1.525 \\
\hline & Carbon. & SON & 6 & 7 & 72 & 0.6694 & 1.44 & 0.880 & 0.887 & 0.895 & 0.916 & 0.893 & 2.608 & 0.798 & 1.754 \\
\hline & Urban & DJF & 33 & 176 & 777 & 0.614 & 1.36 & 0.861 & 0.882 & 0.882 & 0.885 & 0.868 & 1.598 & 1.064 & 1.524 \\
\hline & Urban & MAM & 40 & 230 & 810 & 0.6502 & 1.42 & 0.894 & 0.910 & 0.916 & 0.921 & 0.898 & 2.903 & 1.125 & 1.575 \\
\hline & Urban & JJA & 16 & 66 & 149 & 0.617 & 1.45 & 0.922 & 0.942 & 0.948 & 0.916 & 0.876 & 4.456 & 0.403 & 0.826 \\
\hline & Urban & SON & 26 & 117 & 811 & 0.5993 & 1.37 & 0.893 & 0.903 & 0.900 & 0.897 & 0.874 & 1.722 & 0.843 & 1.260 \\
\hline \multirow{13}{*}{ XiangHe } & Dust & MAM & 9 & 81 & 101 & 1.1211 & 0.08 & 0.884 & 0.908 & 0.927 & 0.941 & 0.959 & 2.847 & 1.933 & 1.932 \\
\hline & Mixt. & DJF & 21 & 140 & 587 & 0.8295 & 0.91 & 0.882 & 0.902 & 0.917 & 0.918 & 0.907 & 3.130 & 0.217 & 1.257 \\
\hline & Mixt. & MAM & 103 & 840 & 2459 & 0.8442 & 0.82 & 0.903 & 0.918 & 0.932 & 0.939 & 0.922 & 3.640 & 0.279 & 1.233 \\
\hline & Mixt. & JJA & 13 & 69 & 162 & 0.863 & 0.84 & 0.908 & 0.923 & 0.927 & 0.911 & 0.871 & 2.120 & -0.554 & 0.623 \\
\hline & Mixt. & SON & 20 & 128 & 748 & 0.7105 & 0.96 & 0.892 & 0.902 & 0.907 & 0.923 & 0.901 & 2.018 & 0.265 & 0.877 \\
\hline & Carbon. & DJF & 39 & 208 & 773 & 0.8137 & 1.35 & 0.878 & 0.898 & 0.914 & 0.919 & 0.905 & 3.636 & 0.857 & 1.793 \\
\hline & Carbon. & MAM & 12 & 37 & 94 & 0.7896 & 1.41 & 0.903 & 0.913 & 0.929 & 0.945 & 0.929 & 5.004 & 0.605 & 1.895 \\
\hline & Carbon. & JJA & 5 & 19 & 33 & 0.9614 & 1.36 & 0.927 & 0.942 & 0.961 & 0.957 & 0.937 & 6.377 & 0.291 & 1.606 \\
\hline & Carbon. & SON & 11 & 30 & 124 & 0.6445 & 1.29 & 0.890 & 0.902 & 0.914 & 0.941 & 0.921 & 2.904 & 0.355 & 1.713 \\
\hline & Urban & DJF & 13 & 82 & 338 & 0.6676 & 1.36 & 0.903 & 0.923 & 0.930 & 0.932 & 0.921 & 2.973 & 0.976 & 1.591 \\
\hline & Urban & MAM & 31 & 212 & 688 & 0.6442 & 1.41 & 0.912 & 0.924 & 0.934 & 0.942 & 0.920 & 3.507 & 0.605 & 1.627 \\
\hline & Urban & JJA & 25 & 104 & 308 & 0.7741 & 1.43 & 0.912 & 0.925 & 0.934 & 0.930 & 0.905 & 5.030 & 0.705 & 1.132 \\
\hline & Urban & SON & 24 & 144 & 738 & 0.5724 & 1.33 & 0.904 & 0.915 & 0.916 & 0.924 & 0.902 & 2.142 & 0.507 & 1.396 \\
\hline \multicolumn{16}{|l|}{ Japan } \\
\hline \multirow[t]{2}{*}{ Osaka } & Mixt. & MAM & 31 & 182 & 283 & 0.6238 & 0.82 & 0.902 & 0.913 & 0.927 & 0.930 & 0.893 & 3.831 & -0.416 & 0.770 \\
\hline & Urban & MAM & 24 & 117 & 207 & 0.5645 & 1.48 & 0.908 & 0.917 & 0.922 & 0.908 & 0.864 & 2.757 & 0.665 & 0.983 \\
\hline \multirow[t]{2}{*}{ Shirahama } & Mixt. & MAM & 16 & 53 & 79 & 0.5818 & 0.81 & 0.910 & 0.926 & 0.941 & 0.927 & 0.863 & 4.850 & -1.637 & 1.079 \\
\hline & Urban & MAM & 12 & 50 & 80 & 0.5342 & 1.43 & 0.907 & 0.919 & 0.920 & 0.917 & 0.882 & 1.922 & 0.875 & 1.327 \\
\hline Northern In & & & & & & & & & & & & & & & \\
\hline
\end{tabular}

Page 9 of 10 


\begin{tabular}{|c|c|c|c|c|c|c|c|c|c|c|c|c|c|c|c|}
\hline \multirow{11}{*}{ Kanpur } & Dust & MAM & 19 & 143 & 181 & 0.8901 & 0.12 & 0.873 & 0.884 & 0.902 & 0.952 & 0.971 & 2.120 & 2.798 & 3.016 \\
\hline & Dust & JJA & 8 & 66 & 26 & 0.8393 & 0.08 & 0.889 & 0.903 & 0.915 & 0.958 & 0.974 & 1.856 & 2.385 & 2.832 \\
\hline & Mixt. & DJF & 22 & 175 & 790 & 0.5799 & 0.99 & 0.883 & 0.893 & 0.901 & 0.908 & 0.856 & 2.303 & -0.249 & 0.697 \\
\hline & Mixt. & MAM & 262 & 2025 & 5087 & 0.6774 & 0.61 & 0.874 & 0.885 & 0.895 & 0.929 & 0.912 & 1.875 & 0.606 & 1.839 \\
\hline & Mixt. & JJA & 39 & 290 & 326 & 0.6765 & 0.48 & 0.893 & 0.908 & 0.920 & 0.958 & 0.927 & 2.556 & -0.760 & 1.973 \\
\hline & Mixt. & SON & 22 & 154 & 545 & 0.5883 & 0.88 & 0.884 & 0.897 & 0.900 & 0.934 & 0.893 & 1.592 & -0.524 & 0.983 \\
\hline & Carbon. & DJF & 25 & 105 & 432 & 0.8159 & 1.38 & 0.915 & 0.914 & 0.925 & 0.931 & 0.912 & 3.570 & 0.581 & 1.155 \\
\hline & Carbon. & SON & 24 & 120 & 583 & 0.9447 & 1.35 & 0.904 & 0.909 & 0.921 & 0.932 & 0.913 & 2.857 & 0.806 & 1.483 \\
\hline & Urban & DJF & 29 & 151 & 1054 & 0.5419 & 1.41 & 0.892 & 0.894 & 0.895 & 0.913 & 0.869 & 1.571 & 0.283 & 1.101 \\
\hline & Urban & MAM & 16 & 132 & 296 & 0.7101 & 1.31 & 0.887 & 0.893 & 0.891 & 0.907 & 0.865 & 1.080 & 0.388 & 0.903 \\
\hline & Urban & SON & 29 & 226 & 1094 & 0.5904 & 1.38 & 0.898 & 0.904 & 0.908 & 0.910 & 0.863 & 1.977 & 0.300 & 1.066 \\
\hline \multirow{8}{*}{ Jaipur } & Dust & MAM & 21 & 157 & 98 & 0.5836 & 0.15 & 0.881 & 0.896 & 0.914 & 0.943 & 0.975 & 3.023 & 3.844 & 3.481 \\
\hline & Dust & JJA & 6 & 56 & 12 & 0.5176 & 0.14 & 0.894 & 0.911 & 0.926 & 0.959 & 0.977 & 2.685 & 3.336 & 3.471 \\
\hline & Mixt. & DJF & 20 & 131 & 380 & 0.5602 & 0.93 & 0.869 & 0.877 & 0.881 & 0.919 & 0.890 & 1.320 & 0.152 & 1.432 \\
\hline & Mixt. & MAM & 78 & 590 & 540 & 0.5304 & 0.55 & 0.881 & 0.893 & 0.900 & 0.933 & 0.916 & 1.837 & 0.238 & 1.792 \\
\hline & Mixt. & JJA & 23 & 148 & 71 & 0.5391 & 0.46 & 0.886 & 0.905 & 0.916 & 0.940 & 0.926 & 2.142 & 0.346 & 1.719 \\
\hline & Mixt. & SON & 19 & 123 & 436 & 0.5315 & 0.81 & 0.874 & 0.886 & 0.885 & 0.923 & 0.878 & 0.840 & -0.267 & 1.062 \\
\hline & Urban & DJF & 22 & 126 & 452 & 0.5308 & 1.34 & 0.877 & 0.881 & 0.874 & 0.906 & 0.889 & 0.760 & 0.943 & 1.519 \\
\hline & Urban & SON & 7 & 36 & 158 & 0.5948 & 1.29 & 0.868 & 0.869 & 0.863 & 0.918 & 0.923 & 0.813 & 1.879 & 2.037 \\
\hline \multirow{8}{*}{ Gandhi_College } & Mixt. & DJF & 6 & 52 & 165 & 0.5102 & 0.97 & 0.865 & 0.871 & 0.877 & 0.927 & 0.868 & 1.689 & -0.690 & 1.150 \\
\hline & Mixt. & MAM & 200 & 1390 & 4099 & 0.7896 & 0.76 & 0.874 & 0.882 & 0.888 & 0.911 & 0.892 & 1.515 & 0.731 & 1.584 \\
\hline & Mixt. & JJA & 32 & 163 & 352 & 0.7924 & 0.6 & 0.886 & 0.896 & 0.903 & 0.941 & 0.923 & 1.720 & 0.023 & 1.691 \\
\hline & Mixt. & SON & 6 & 30 & 101 & 0.5398 & 0.63 & 0.901 & 0.922 & 0.940 & 0.950 & 0.884 & 3.973 & -2.412 & 0.619 \\
\hline & Carbon. & SON & 8 & 24 & 162 & 0.9048 & 1.39 & 0.917 & 0.919 & 0.928 & 0.938 & 0.925 & 2.807 & 1.059 & 1.412 \\
\hline & Urban & DJF & 7 & 56 & 264 & 0.5381 & 1.42 & 0.894 & 0.900 & 0.898 & 0.905 & 0.869 & 1.328 & 0.684 & 1.118 \\
\hline & Urban & MAM & 26 & 140 & 495 & 0.7505 & 1.37 & 0.890 & 0.890 & 0.884 & 0.896 & 0.882 & 0.844 & 1.524 & 1.556 \\
\hline & Urban & SON & 9 & 59 & 230 & 0.5532 & 1.38 & 0.893 & 0.905 & 0.911 & 0.903 & 0.836 & 2.302 & -0.153 & 0.817 \\
\hline \multicolumn{16}{|c|}{ Northern Australia } \\
\hline \multirow[t]{2}{*}{ Jabiru } & Carbon. & SON & 10 & 43 & 56 & 0.5487 & 1.7 & 0.891 & 0.898 & 0.913 & 0.895 & 0.872 & 4.855 & 1.534 & 1.594 \\
\hline & Urban & SON & 13 & 56 & 101 & 0.499 & 1.71 & 0.890 & 0.890 & 0.891 & 0.892 & 0.880 & 1.844 & 1.688 & 1.519 \\
\hline \multirow[t]{2}{*}{ Lake_Argyle } & Carbon. & SON & 33 & 174 & 496 & 0.6524 & 1.59 & 0.872 & 0.872 & 0.876 & 0.883 & 0.870 & 2.131 & 1.731 & 1.350 \\
\hline & Urban & SON & 21 & 95 & 230 & 0.4725 & 1.65 & 0.857 & 0.862 & 0.864 & 0.881 & 0.862 & 1.862 & 2.008 & 1.628 \\
\hline
\end{tabular}

